Pacific Journal of Mathematic 


\title{
IDENTIFICATION SPACES AND UNIQUE UNIFORMITY
}

\author{
RICHARD H. WARREN
}

\begin{abstract}
Properties of $T_{0}$-identification spaces and uniform identification spaces are used to obtain necessary and sufficient conditions for topological spaces to have a unique compatible uniformity.
\end{abstract}

1. Introduction. The major results in this paper are five characterizations of completely regular spaces with a unique compatible uniformity. All prior results of this type assume the space to be Tychonoff, i.e., completely regular and Hausdorff. In our approach we introduce a uniform identification space and develop some of its properties in order to demonstrate a $1-1$ correspondence between the family of compatible uniformities on a completely regular space and the family of compatible uniformities on its $T_{0}$-identification space.

Section 2 contains background on $T_{0}$-identification spaces and several new features of such spaces which we use later in the paper. In $\S 3$ we present the main aspects of uniform identification spaces which lead to the order isomorphism in Theorem 3.5. In $\S 4$, which contains the major theorem of the paper, we sketch the development of five characterizations of Tychonoff spaces with a unique compatible uniformity and then prove that each of these characterizations has a parallel for completely regular spaces.

2. $T_{0}$-identification spaces. In $1936 \mathrm{M} . \mathrm{H}$. Stone proved that every topological space can be made into a $T_{0}$-space by identifying points with the same closure. A complete statement of Stone's work, with additional properties not included in Stone's paper, can be found in Theorem 14.2 in [8]. Briefly, given a topological space $(X, \mathscr{T})$, define $x \sim y$ iff $\overline{\{x\}}=\overline{\{y\}}$. Then $\sim$ is an equivalence relation on $X$ and the quotient space $(Y, \mathscr{V})$ is a $T_{0}$-space. For $x \in X$, let $D_{x}$ be the member of $Y$ containing $x$. Then $f: X \rightarrow Y$ by $f(x)=D_{x}$ is a continuous, open and closed map onto $Y$.

Throughout this section $(Y, \mathscr{Y})$ will be the $T_{0}$-identification space of $(X, \mathscr{T})$, and $D_{x}$ and $f$ will be as designated in the two preceding sentences.

A topological space $(X, \mathscr{T})$ is said to be $C$-embedded (in the terminology of [3], normally embedded) in the space $(Z, \mathscr{C})$ if every real-valued, continuous function on $X$ has a continuous extension to $Z$, possibly through a homeomorphism of $(X, \mathscr{T})$ onto a subspace of $(Z, \mathscr{Q})$. Such a homeomorphism is called an embedding of $(X, \mathscr{T})$ 
into $(Z, \mathscr{U})$. Also, $(X, \mathscr{T})$ is said to be densely embedded in $(Z, \mathscr{U})$ if there is an embedding $f$ of $(X, \mathscr{T})$ into $(Z, \mathscr{U})$ such that $f(X)$ is dense in $Z$.

TheOREM 2.1. ( $Y, \mathscr{V})$ is densely embedded in $(X, \mathscr{T})$.

Proof. Let $W$ be a subset of $X$ containing exactly one element of each equivalence class. Let $g: W \rightarrow Y$ by $g(w)=D_{w}$. Clearly $g$ is $1-1$ and onto. To verify that $g$ is continuous, let $G \in \mathscr{V}$. Then $f^{-1}(G) \in \mathscr{T}$ and thus $f^{-1}(G) \cap W$ is open in $W$. Since $f^{-1}(G)=$ $\cup\left\{D_{x}: D_{x} \in G\right\}$, it follows that $g^{-1}(G)=f^{-1}(G) \cap W$. To verify that $g^{-1}$ is continuous, note that if $H \in \mathscr{T}$, then $H=\cup\left\{D_{x}: x \in H\right\}$. Hence $g(H \cap W)=f(H) \in \mathscr{V}$.

To see that $\bar{W}=X$, let $x \in X$. Then $x \in D_{w}$ for some $w \in W$. Thus $w$ is in every neighborhood of $x$. Therefore $x \in \bar{W}$.

Corollary 2.1. ( $Y, \mathscr{T})$ is C-embedded in $(X, \mathscr{T})$.

Proof. Let $g$ be the homeomorphism in the previous proof. Given a real-valued, continuous map $h$ on $(Y, \mathscr{V})$, then $h \circ f$ is a real-valued, continuous map on $\left(X, \mathscr{S}^{\prime}\right)$ which extends $h \circ g$.

Lemma 2.1. If $g: X \rightarrow S$ is continuous and $(S, \mathscr{C})$ is a $T_{0}$-space, then $g(x)=g(y)$ whenever $y \in D_{x}$.

Proof. Suppose $y \in D_{x}$ and $g(x) \neq g(y)$. Since $\left(S, \mathscr{C}_{6}\right)$ is $T_{0}$, there is $G \in \mathscr{U}$ such that $G \cap\{g(x), g(y)\}$ is a singleton. Thus the open set $g^{-1}(G)$ contains exactly one of the points $x, y$ which contradicts the equivalence relation determining $D_{x}$.

THEOREM 2.2. ( $Y, \mathscr{Y})$ has property $\left({ }^{*}\right)$ iff $(X, \mathscr{T})$ does.

$\left({ }^{*}\right)$ For every real-valued, continuous function $g$ defined on the space, $g^{-1}(\{r: r \geqq 1\})$ or $g^{-1}(\{r: r \leqq 0\})$ is compact.

Proof. Let $g$ be a real-valued, continuous function defined on $X$. As a result of Lemma 2.1, we may define a real-valued function $h$ on $Y$ by $h\left(D_{x}\right)=g(x)$ for each $D_{x} \in Y$. Hence $g=h \circ f$. It is easy to show that $h$ is continuous and if $C$ is a compact subset of $Y$, then $f^{-1}(C)$ is a compact subset of $X$. Therefore, if $h$ has property $(*)$, then $g$ does also.

The proof of the converse is straightforward. 
THEOREM 2.3. If $X$ is a set, $Y$ is a partition of $X$ and $\mathscr{V}$ is a $T_{0}$-topology on $Y$, then there is a unique topology $\mathscr{T}$ on $X$ such that $(Y, \mathscr{V})$ is the $T_{0}$-identification space of $(X, \mathscr{T})$.

Proof. Since $Y$ is a collection of disjoint subsets of $X$ which covers $X$, for each $x \in X$ there is exactly one $D_{x} \in Y$ such that $x \in D_{x}$. Let $f: X \rightarrow Y$ by $f(x)=D_{x}$. By Theorem 10.10 in [8] the family $\mathscr{J}^{-}=\left\{f^{-1}(B): B \in \mathscr{Y}\right\}$ is the weakest topology on $X$ such that $f$ is continuous. We shall show that $(Y, \mathscr{C})$ is the $T_{0}$-identification of $(X, \mathscr{T})$.

Let $x, y \in X$. If $y \in D_{x}$ and $x \in f^{-1}(B)$ where $B \in \mathscr{V}$, then since $f^{-1}(B)=\cup\left\{D_{x}: D_{x} \in B\right\}$, it follows that $y \in f^{-1}(B)$, i.e., each member of $D_{x}$ is in every open subset of $X$ which contains $x$. On the other hand, if $y \notin D_{x}$, then $D_{y} \cap D_{x}=\varnothing$. Since $(Y, \mathscr{Y})$ is $T_{0}$, there exists $B \in \mathscr{Y}$ which contains $D_{y}$ or $D_{x}$, but not both. Hence $f^{-1}(B)$ contains $x$ or $y$, but not both. Therefore the members of $Y$ are exactly the classes which are determined by the equivalence relation on $X$ where $x \approx y$ iff $\overline{\{x\}}=\overline{\{y\}}$.

Let $\mathscr{U}$ be the quotient topology on $Y$ determined by $f$. Since $\mathscr{Z}$ is the strongest topology on $Y$ such that $f$ is continuous, $\mathscr{C} \subset \mathscr{W}$. If $G \in \mathscr{C}$, then $f^{-1}(G) \in \mathscr{S}$ and there is $B \in \mathscr{V}$ such that $f^{-1}(B)=f^{-1}(G)$. Since $f$ is onto, $B=f\left(f^{-1}(B)\right)=f\left(f^{-1}(G)\right)=G$.

To see that $\mathscr{T}$ is unique, let $\mathscr{S}$ be a topology on $X$ such that $(Y, \mathscr{V})$ is the $T_{0}$-identification of $(X, \mathscr{S})$. Since $\mathscr{T}$ is the weakest topology on $X$ such that $f$ is continuous, $\mathscr{S} \subset \mathscr{S}$. Suppose $S \in \mathscr{S} \backslash \mathscr{T}$. Since $f$ is an open map, $f(S) \in \mathscr{V}$. So $f^{-1}(f(S)) \in \mathscr{T}$ and there is $t \in f^{-1}(f(S)) \backslash S$. Now $t \in D_{s}$ for some $s \in S$. Thus $s$ is a member of a set in $\mathscr{S}$ not containing $t$, which contradicts the equivalence relation $\approx$

Theorem 2.4. Let $(X, \mathscr{T})$ be a subspace of $(S, \mathscr{S})$ whose $T_{0^{-}}$ identification space is $(T, \mathscr{C})$. If $(Y, \mathscr{C})$ is $C$-embedded in $(T, \mathscr{U})$, then $(X, \mathscr{T})$ is C-embedded in $(S, \mathscr{S})$.

Proof. Let $g$ be a continuous, real-valued function on $X$. As a result of Lemma 2.1, we may define a real-valued function $h$ on $Y$ by $h\left(D_{x}\right)=g(x)$ for each $D_{x} \in Y$. Hence $g=h \circ f$ and $h$ is continuous. By assumption $h$ has a continuous extension $k$ to $T$. Let $e: S \rightarrow T$ be the quotient map $e(s)=[s]$ where $[s]$ is the equivalence class containing $s$. Then $k \circ e$ is a continuous extension of $g$ to $S$.

TheOREM 2.5. Let $(Y, \mathscr{V})$ be a dense subspace of the $T_{0}$-space $(T, \mathscr{C})$. Then there is a topological space $(S, \mathscr{S})$ such that $(T, \mathscr{U})$ 
is the $T_{0}$-identification of $(S, \mathscr{S})$ and $(X, \mathscr{T})$ is densely embedded in $(S, \mathscr{S})$. Furthermore, if $(X, \mathscr{T})$ is $C$-embedded in $(S, \mathscr{S})$, then $(Y, \mathscr{V})$ is $C$-embedded in $(T, \mathscr{C})$.

Proof. Let $S=X \cup(T \backslash Y)$, so without loss of generality we may assume $T \cap X=\varnothing$. For each open subset $A$ of $T$ form $A^{*}=$ $\cup\left\{D_{x}: D_{x} \in A \cap Y\right\} \cup A \backslash Y$. Then $\left\{A^{*}: A \in \mathscr{U}\right\}$ is a topology on $S$. As usual, define $x \approx y$ for $x, y \in S$ iff $\overline{\{x\}^{S}}=\overline{\{y\}^{S}}$. Note that when $x$ and $y$ are distinct points in $S$, then $x \approx y$ iff $x, y \in X$ and $x \sim y$ in $X$. Thus $\approx$ determines the members of $T$, with the identification of $\{t\}$ with $t$ whenever $t \in T \backslash Y$. It is easy to show that the quotient topology on $T$ agrees with $\mathscr{C}$ and that $(X, \mathscr{T})$ is a dense subspace of $(S, \mathscr{S})$.

Let $h$ be a real-valued, continuous function on $Y$. Then $h \circ f$ is a real-valued, continuous function on $X$, and thus has a continuous extension $j$ to $S$. As a result of Lemma 2.1, we may define a realvalued function $k$ on $T$ by $k\left(D_{x}\right)=j(x)$ for $D_{x} \in Y$ and $k(t)=j(t)$ for $t \in T \backslash Y$. Let $e: S \rightarrow T$ be the quotient map. Then $j=k \circ e, k$ is continuous and $\left.k\right|_{Y}=h$.

Let $C^{*}(X)$ be the set of bounded, real-valued, continuous functions on $X$ and let $C^{*}(X)$ have the topology of uniform convergence. By $A(X)$ we denote the subset of $C^{*}(X)$ consisting of those functions which are constant on the complement of some compact set in $(X, \mathscr{T})$.

THEOREM 2.6. Let $(Y, \mathscr{Y})$ be the $T_{0}$-identification space of $(X, \mathscr{T})$. Then $A(X)$ is dense in $C^{*}(X)$ iff $A(Y)$ is dense in $C^{*}(Y)$.

Proof. $\quad(\Leftarrow)$ Let $g \in C^{*}(X)$ and $\varepsilon>0$. As a result of Lemma 2.1, we may define a real-valued function $h$ on $Y$ by $h\left(D_{x}\right)=g(x)$ for each $D_{x} \in Y$. Then $g=h \circ f$ and $h \in C^{*}(Y)$. Since $A(Y)$ is dense in $C^{*}(Y)$, there is a continuous function $k$ which is constant on the complement of a compact set $C$ in $Y$ and satisfies $\left|k\left(D_{x}\right)-h\left(D_{x}\right)\right|<\varepsilon$ for each $D_{x} \in Y$. Then $f^{-1}(C)$ is compact in $X$ and $k \circ f$ is constant on the complement of $f^{-1}(C)$. Also $|k(f(x))-g(x)|<\varepsilon$ for each $x \in X$.

$\Leftrightarrow$ Let $h \in C^{*}(Y)$ and $\varepsilon>0$. Then $h \circ f \in C^{*}(X)$. Since $A(X)$ is dense in $C^{*}(X)$, there is a continuous function $g$ which is constant on the complement of a compact set $K$ in $X$ and satisfies $\mid h(f(x))$ $g(x) \mid<\varepsilon$ for each $x \in X$. As a result of Lemma 2.1, we may define a real-valued function $k$ on $Y$ by $k\left(D_{x}\right)=g(x)$ for each $D_{x} \in Y$. Then $g=k \circ f$ and $k$ is constant on the complement of the compact set $f(K)$ in $Y$. Also $\left|h\left(D_{x}\right)-k\left(D_{x}\right)\right|<\varepsilon$ for each $D_{x} \in X$. 
3. Uniform identification spaces. For the definitions of a uniform space and a proximity space, see [8]. Recall that if $U, V \subset$ $X \times X$, then $U \circ V=\{(x, y):(x, t) \in U$ and $(t, y) \in V$ for some $t\}$ and $U(x)=\{y:(x, y) \in U\}$. If $(X, \mathscr{H})$ is a uniform space and $Z$ is a set with $h: X \rightarrow Z$ a map onto $Z$, then the quotient uniformity induced on $Z$ by $h$ is $\{A \subset Z \times Z$ : there is $H \in \mathscr{X}$ such that $(x, y) \in H$ implies $(h(x), h(y)) \in A\}$, which is the largest uniformity on $Z$ such that $h$ is uniformly continuous, see $[9$, p. 255]. It is easily verified that the quotient uniformity is $\{g(H): H \in \mathscr{C}\}$ where $g(H)=\{(h(a), h(b))$ : $(a, b) \in H\}$.

Let $(X, \mathscr{C})$ be a uniform space. For $x, y \in X$, define $x \sim y$ iff $y \in H(x)$ for each $H \in \mathscr{H}$. Then $\sim$ is an equivalence relation on $X$. Throughout this section $Y$ is the set of equivalence classes, $D_{x}$ is the member of $Y$ containing $x, f: X \rightarrow Y$ by $f(x)=D_{x}$ and $\mathscr{K}$ is the quotient uniformity on $Y$ induced by $f$. Also, $(Y, \mathscr{K})$ is called the uniform identification space of $(X, \mathscr{H})$.

Lemma 3.1. ( $Y, \mathscr{K})$ is a separated uniform space.

Proof. Suppose $D_{x}$ and $D_{y}$ are distinct equivalence classes. Then there exists $H \in \mathscr{C}$ such that $H(x) \cap H(y)=\varnothing$. Since $D_{y} \subset H(y)$, $H(x) \cap D_{y}=\varnothing$. Choose $F \in \mathscr{C}$ such that $F \circ F \subset H$. If there exists $a \in D_{x}$ and $b \in D_{y}$ such that $(a, b) \in F$, then $(x, a) \in F$, since $x \in D_{x} \subset$ $F(x)$. Hence $(x, b) \in F \circ F$, and thus $b \in H(x)$ which is a contradiction. We conclude that $\left(D_{x} \times D_{y}\right) \cap F=\varnothing$. Therefore $\left(D_{x}, D_{y}\right) \notin g(F) \in \mathscr{K}$.

Corollary 3.1. If $(X, \mathscr{H})$ is a uniform space, then $\cap\{H: H \in$ $\mathscr{H}\}=\bigcup\left\{D_{x} \times D_{x}: x \in X\right\}$.

Proof. Using $F$ in the proof of Lemma 3.1, it follows that $\cap\{H: H \in \mathscr{H}\} \subset \cup\left\{D_{x} \times D_{x}: x \in X\right\}$. The other containment is a result of $D_{x} \subset H(x)$ for each $H \in \mathscr{H}$.

THEOREM 3.1. Let $(X, \mathscr{H})$ be a uniform space, let $\delta$ be the proximity on $X$ induced by $\mathscr{H}$ and let $\alpha$ be the proximity on $Y$ induced by $\mathscr{K}$. Then the following diagram commutes.

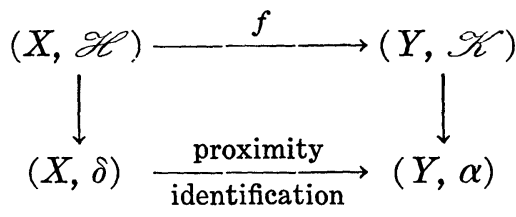

Proof. In $(X, \delta)$, the equivalence classes are determined by the 
relation $x \approx y$ iff $x \delta y$ [9, p. 276]. Since $x \delta y$ iff $y \in H(x)$ for each $H \in \mathscr{H}$ iff $x \sim y$, it follows that the same members are determined for $Y$ by either path. Since the proximity induced by a quotient uniformity is the quotient proximity of the induced proximity [9, p. 276], the diagram commutes.

CoRollary 3.2. In addition, let $\mathscr{T}$ be the topology on $X$ induced by $\delta$ and let $\mathscr{V}$ be the topology on $Y$ induced by $\alpha$. Then the following diagram commutes.

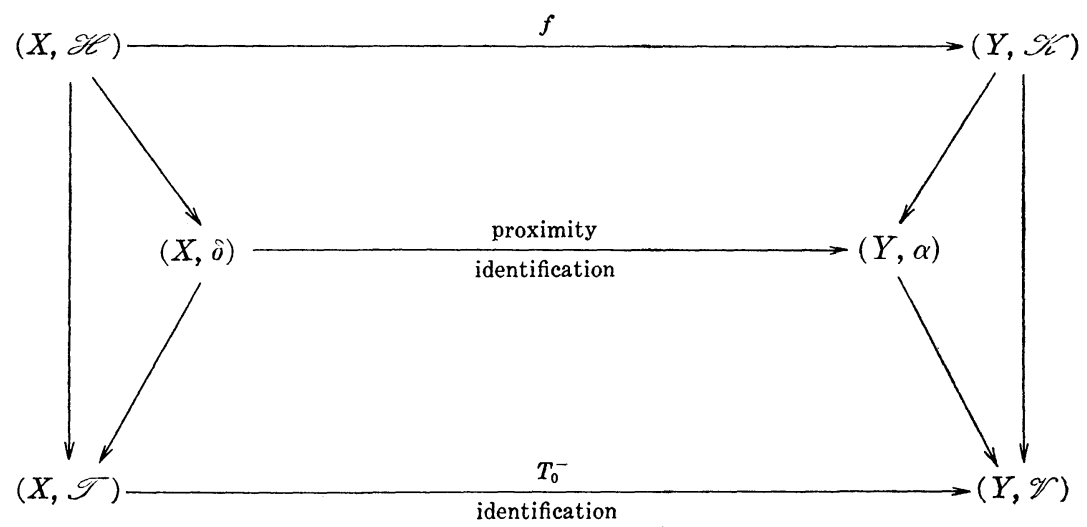

Proof. The topology induced by a uniformity is the induced topology of the induced proximity [8, Theorem 21.15]. Starting with $(X, \delta)$, it is known that the paths are equivalent [9, p. 276].

Lemma 3.2. Let $(X, \mathscr{H})$ be a uniform space and let $H \in \mathscr{X}$. Then there is $E \in \mathscr{H}$ such that $E \subset H$ and $E=\cup\left\{D_{x} \times D_{y}:(x, y) \in E\right\}$.

Proof. Find symmetric $G \in \mathscr{Y}$ such that $G \circ G \subset H$. Note that $G \subset G \circ G$. Then find $F \in \mathscr{Y}$ such that $F \circ F \subset G$. If $(r, s) \notin H$, then $H(r) \cap\{s\}=\varnothing$. If there exists $t \in G(r) \cap G(s)$, then $(r, t)$ and $(s, t)$ are in $G$. Since $G$ is symmetric, $(r, s) \in G \circ G$, which is a contradiction. Thus $G(r) \cap G(s)=\varnothing$. As in the proof of Lemma 3.1 we can conclude that $\left(D_{r} \times D_{s}\right) \cap F=\varnothing$. Set $E=\cup\left\{D_{x} \times D_{y}:(x, y) \in F\right\}$. Since $(x, y) \in D_{x} \times D_{y}$, if follows that $F \subset E$ and hence, $E \in \mathscr{Z}$. If $(a, b) \in E$, then $\left(D_{a} \times D_{b}\right) \cap F \neq \varnothing$, and from the above work $(a, b) \in H$. Clearly, $E=\cup\left\{D_{x} \times D_{y}:(x, y) \in E\right\}$.

THEOREM 3.2. (Y, $\mathscr{K})$ is uniformly isomorphic to a uniform subspace of $(X, \mathscr{H})$.

Proof. Let $S$ be a subset of $X$ consisting of exactly one point 
from each equivalence class in $Y$. The relative uniformity on $S$ is $\{H \cap(S \times S): H \in \mathscr{X}\}$. Consider the map $d: S \rightarrow Y$ by $d(s)=D_{s}$. Clearly, $d$ is $1-1$ and onto. To verify that $d$ is uniformly continuous, let $K \in \mathscr{K}$. Then there is $H \in \mathscr{C}$ such that $f(H)=K$. If $(x, y) \in H \cap(S \times S)$, then $(d(x), d(y))=(f(x), f(y)) \in f(H)$. To verify that $d^{-1}$ is uniformly continuous, let $H \in \mathscr{X}$ and consider $E \cap(S \times S)$ where $E$ is the entourage in $\mathscr{C}$ guaranteed by Lemma 3.2. If $\left(D_{x}, D_{y}\right) \in f(E)$ which is in $\mathscr{K}$, then $\left(d^{-1}\left(D_{x}\right), d^{-1}\left(D_{y}\right)\right) \in f^{-1}(f(E)) \cap$ $(S \times S)=E \cap(S \times S) \subset H \cap(S \times S)$.

THEOREM 3.3. Let $(Y, \mathscr{K})$ be the uniform identification space of $(X, \mathscr{H})$. Then $\mathscr{F}=\left\{g^{-1}(K): K \in \mathscr{K}\right\}$ is a base for $\mathscr{H}$, where $g^{-1}(K)=\{(a, b) \in X \times X:(f(a), f(b)) \in K\}$.

Proof. By Theorem 20.21 in [8], $\mathscr{F}$ is a subbase for the weakest uniformity $\mathscr{G}$ on $X$ such that $f$ is uniformly continuous. Thus $\mathscr{G} \subset \mathscr{H}$. To verify that $\mathscr{F}$ is a base, note that $g^{-1}(K)=\cup\left\{D_{x} \times D_{y}\right.$ : $\left.\left(D_{x}, D_{y}\right) \in K\right\}$ and $D_{x} \times D_{y}=D_{r} \times D_{s}$ or $\left(D_{x} \times D_{y}\right) \cap\left(D_{r} \times D_{s}\right)=\varnothing$, from which it follows that $g^{-1}(K) \cap g^{-1}(L)=g^{-1}(K \cap L)$. If $H \in \mathscr{H}$, then let $E \in \mathscr{H}$ be the entourage guaranteed by Lemma 3.2. Therefore $E=g^{-1}(g(E))$ and $g(E) \in \mathscr{K}$. Hence $\mathscr{K} \subset \mathscr{G}$.

THEOREM 3.4. Let $X$ be a set, let $Y$ be a partition of $X$ and let $\mathscr{K}$ be a separated uniformity on $Y$. Then there is a uniformity $\mathscr{H}$ on $X$ such that $(Y, \mathscr{K})$ is the uniform identification space of $(X, \mathscr{H})$.

Proof. Since $Y$ is a collection of disjoint subsets of $X$ which covers $X$, for each $x \in X$ there is exactly one member $D_{x}$ of $Y$ such that $x \in D_{x}$. Let $f: X \rightarrow Y$ by $f(x)=D_{x}$. By Theorem 20.21 in [8], the family $\mathscr{F}=\left\{g^{-1}(K): K \in \mathscr{K}\right\}$ is a subbase for the weakest uniformity $\mathscr{H}$ on $X$ such that $f$ is uniformly continuous. Here $g^{-1}(K)=$ $\{(a, b) \in X \times X:(f(a), f(b)) \in K\}$. We shall show that $(Y, \mathscr{K})$ is the uniform identification space of $(X, \mathscr{H})$.

If $y \in D_{x}$ and $K \in \mathscr{K}$, then $\left(D_{x}, D_{x}\right) \in K$ and hence $y \in g^{-1}(K)(x)$. If $y \notin D_{x}$, then since $\mathscr{K}$ is separated, there is some $K_{0} \in \mathscr{K}$ such that $\left(D_{x}, D_{y}\right) \notin K_{0}$. Thus $y \notin g^{-1}\left(K_{0}\right)(x)$. We conclude that the members of $Y$ are exactly the classes which are determined by the equivalence relation on $X$ where $x \sim y$ iff $y \in H(x)$ for each $H \in \mathscr{H}$.

Let $\mathscr{L}$ be the quotient uniformity on $Y$ induced by $f$. Since $f$ is uniformly continuous with respect to $\mathscr{K}$ and $\mathscr{L}, \mathscr{K} \subset \mathscr{L}$. On the other hand, if $L \in \mathscr{L}$, then there is $H \in \mathscr{\mathscr { C }}$ such that $h(H)=L$, where $h(H)=\{(f(r), f(s)):(r, s) \in H\}$. Since $\mathscr{F}$ is a base for $\mathscr{H}$, there is $F \in \mathscr{F}$ such that $F \subset H$. Then there is $K \in \mathscr{K}$ such that 
$g^{-1}(K)=F$. Thus $K \subset L$ and therefore $L \in \mathscr{K}$.

THEOREM 3.5. Let $(X, \mathscr{T})$ be a topological space and let $(Y, \mathscr{V})$ be its $T_{0}$-identification space. Let $\Theta$ be the family of all uniformities on $X$ compatible with $\mathscr{T}$ and let $\Omega$ be the family of all uniformities on $Y$ compatible with $\mathscr{V}$. Then $\Theta$ and $\Omega$ are order isomorphic.

Proof. By Corollary 3.2 we may define $h: \Theta \rightarrow \Omega$ by $h(\mathscr{H})$ is the identification uniformity of $\mathscr{H}$. As a result of Theorem $3.3, h$ is $1-1$. By Theorem 3.4 in conjunction with Theorem 2.3 and Corollary $3.2, h$ is onto. Since $h(\mathscr{H})$ is a quotient uniformity, it follows that $h$ preserves order. Noting how $h^{-1}(\mathscr{K})$ is formed in the proof of Theorem 3.4 allows us to conclude that $h^{-1}$ preserves order.

It is noted that Theorem 3.5 can also be proved from Theorem 2.1 in [7].

4. Unique compatible uniformity and proximity. Early in the study of uniform spaces it was observed that a compact, completely regular topological space admits exactly one compatible uniformity [8, Theorem 20.38]. Using normally separated sets, Doss [2] characterized Tychonoff spaces which have exactly one compatible uniformity. Later Gál [3] gave two additional characterizations of Tychonoff spaces with this phenomenon. Newns [6] has given a characterization based on the structure of the uniformity. Doss' work is extended to completely regular spaces in Theorem 4.1(d), Gál's work in Theorem 4.1(e) and (f), and Newns' work in Theorem 4.1(b).

In Corollary 2.2 of [4] it is shown that a Tychonoff space has a unique compatible proximity iff it has a unique compatible uniformity. Note that [4] requires a Hausdorff assumption since Corollary 2.2 is based upon the Stone-Čech and Smirnov compactifications. This result is also in [9, p. 277]. We prove in the next theorem that this result is valid without a Hausdorff assumption.

THEOREM 4.1. Let $(X, \mathscr{T})$ be a completely regular topological space. Then the following are equivalent:

(a) There is a unique uniformity on $X$ compatible with $\mathscr{T}$.

(b) There is a unique totally bounded uniformity on $X$ compatible with $\mathscr{T}$.

(c) There is a unique proximity on $X$ compatible with $\mathscr{T}$.

(d) $(X, \mathscr{T})$ has the property that for every real-valued, continuous function $f$ defined on $X,\{x: f(x) \geqq 1\}$ or $\{x: f(x) \leqq 0\}$ is compact. 
(e) $(X, \mathscr{T})$ is C-embedded in every completely regular space containing $(X, \mathscr{T})$ as a dense subspace.

(f) $A(X)$ is dense in $C^{*}(X)$.

Proof. Let $(Y, \mathscr{T})$ be the $T_{0}$-identification space of $(X, \mathscr{T})$.

$(b) \Leftrightarrow(c)$. The implications follow from Theorem 21.28 in [8].

$(\mathrm{a}) \Leftrightarrow(\mathrm{c})$. Since $\mathscr{T}$ and $\mathscr{Y}$ are lattice isomorphic, it follows from [1] that the family of proximities on $X$ compatible with $\mathscr{T}$ is isomorphic to the family of proximities on $Y$ compatible with $\mathscr{Y}$. Hence (b) is equivalent to the existence of a unique proximity on $Y$ compatible with $\mathscr{Y}$. From [4, Corollary 2.2] this is equivalent to the existence of a unique uniformity on $Y$ compatible with $\mathscr{Y}$. By Theorem 3.5 this last statement is equivalent to (a). [2].

$(\mathrm{a}) \Leftrightarrow(\mathrm{d})$. Couple Theorems 3.5 and 2.2 with Doss' Theorem in

(a) $\Leftrightarrow(\mathrm{e})$. Let $X$ be a dense subspace of the completely regular space $S$ whose $T_{0}$-identification space is $T$. Denote the equivalence classes of $S$ by $[s]$ where $s \in S$. If $s \in X$, then we identify the equivalence class $D_{s}$ of $X$ with [s] of $S$. Thus $Y$ is densely embedded in T. It follows from Theorem 3.5 that (a) is equivalent to $Y$ having a unique uniformity, which by Gál's Theorem (v) in [3] implies that $Y$ is $C$-embedded in $T$, and by Theorem $2.4 X$ is $C$-embedded in $S$. On the other hand, (d) implies by Theorem 2.5 that $Y$ is $C$-embedded in every Tychonoff space containing $Y$ as a dense subspace, which by Gál's Theorem (v) in [3], is equivalent to $Y$ having a unique uniformity. in [3].

(a) $\Leftrightarrow(\mathbf{f})$. Couple Theorems 3.5 and 2.6 with Gál's Theorem (iv)

Each of the following three statements is equivalent to Theorem 4.1(d). In the terminology of [2], of any two normally separable sets in $X$, at least one is compact. In the terminology of [5], if $A$ and $B$ are functionally distinguishable subsets of $X$, then $A$ or $B$ is compact. In the terminology of [9], of any two disjoint zero-sets in $X$, at least one is compact.

Comparing Theorem 4.1(d) with Theorem 7.20 in [5], it is noted that locally compact can be deleted from the statement of Theorem 7.20 .

\section{REFERENCES}

1. T. E. Dooher and W. J. Thron, Proximities compatible with a given topology, Dissertationes Math. (Rozprawy Mat.), 71 (1970), 1-38.

2. R. Doss, On uniform spaces with a unique structure, Amer. J. Math., 71 (1949), 19-23.

3. I. S. Gál, Uniformizable spaces with a unique structure, Pacific J. Math., 9 (1959), 1053-1060. 
4. D. R. Johnson and D. A. Mattson, Some applications of non-standard analysis to proximity spaces, Colloq. Math., 34 (1975), 17-24.

5. S. A. Naimpally and B. D. Warrack, Proximity Spaces, Cambridge University Press, London, 1970.

6. W. F. Newns, Uniform spaces with unique structure, Amer. J. Math., 79 (1957), 48-52.

7. E. E. Reed and W. J. Thron, Uniform structures on the lattices of open sets, Math. Nachr., 36 (1968), 237-253.

8. W. J. Thron, Topological Structures, Holt, Rinehart and Winston, New York, 1966.

9. S. Willard, General Topology, Addison-Wesley, Reading, 1970.

Received June 15, 1979 and in revised form December 5, 1979. The author gratefully acknowledges the support of The Institute for Advanced Study, Princeton, NJ where the research was accomplished.

University of Nebraska at Omaha

ОмАна, NE 68182 


\section{PACIFIC JOURNAL OF MATHEMATICS}

\section{EDITORS}

DONALD BABBITT (Managing Editor)

University of California

Los Angeles, CA 90024

HUGo RossI

University of Utah

Salt Lake City, UT 84112

C. C. MOORE and ANDREW OGG

University of California

Berkeley, CA 94720
J. DugundjI

Department of Mathematics

University of Southern California

Los Angeles, CA 90007

R. FinN and J. Milgram

Stanford University

Stanford, CA 94305

\section{ASSOCIATE EDITORS}
R. ARENS
E. F. BECKENBACH
B. H. NeumanN
F. WOLF
K. YOSHIDA

\section{SUPPORTING INSTITUTIONS}

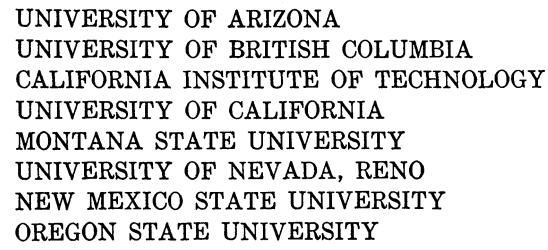

UNIVERSITY OF ARIZONA

UNIVERSITY OF BRITISH COLUMBIA

CALIFORNIA INSTITUTE OF TECHNOLOGY

UNIVERSITY OF CALIFORNIA

MONTANA STATE UNIVERSITY

UNIVERSITY OF NEVADA, RENO

NEW MEXICO STATE UNIVERSITY

OREGON STATE UNIVERSITY

\author{
UNIVERSITY OF OREGON \\ UNIVERSITY OF SOUTHERN CALIFORNIA \\ STANFORD UNIVERSITY \\ UNIVERSITY OF HAWAII \\ UNIVERSITY OF TOKYO \\ UNIVERSITY OF UTAH \\ WASHINGTON STATE UNIVERSITY \\ UNIVERSITY OF WASHINGTON
}

The Supporting Institutions listed above contribute to the cost of publication of this Journal, but they are not owners or publishers and have no responsibility for its content or policies.

Mathematical papers intended for publication in the Pacific Journal of Mathematics should be in typed form or offset-reproduced, (not dittoed), double spaced with large margins. Please do not use built up fractions in the text of the manuscript. However, you may use them in the displayed equations. Underline Greek letters in red, German in green, and script in blue. The first paragraph or two must be capable of being used separately as a synopsis of the entire paper. Please propose a heading for the odd numbered pages of less than 35 characters. Manuscripts, in triplicate, may be sent to any one of the editors. Please classify according to the scheme of Math. Reviews, Index to Vol. 39. Supply name and address of author to whom proofs should be sent. All other communications should be addressed to the managing editor, or Elaine Barth, University of California, Los Angeles, California, 90024.

50 reprints to each author are provided free for each article, only if page charges have been substantially paid. Additional copies may be obtained at cost in multiples of 50 .

The Pacific Journal of Mathematics is issued monthly as of January 1966. Regular subscription rate: $\$ 102.00$ a year (6 Vols., 12 issues). Special rate: $\$ 51.00$ a year to individual members of supporting institutions.

Subscriptions, orders for numbers issued in the last three calendar years, and changes of address shoud be sent to Pacific Journal of Mathematics, P.O. Box 969, Carmel Valley, CA 93924, U.S.A. Old back numbers obtainable from Kraus Per!odicals Co., Route 100, Millwood, NY 10546.

PUBLISHED BY PACIFIC JOURNAL OF MATHEMATICS, A NON-PROFIT CORPORATION

Printed at Kokusai Bunken Insatsusha (International Academic Printing Co., Ltd.). 8-8, 3-chome, Takadanobaba, Shinjuku-ku, Tokyo 160, Japan. 


\section{Pacific Journal of Mathematics}

\section{Vol. 95, No. $2 \quad$ October, 1981}

George E. Andrews, The Rogers-Ramanujan reciprocal and Minc's

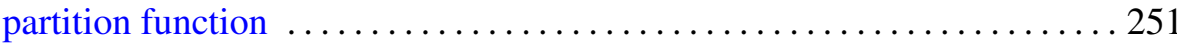

Allan Calder, William H. Julian, Ray Mines, III and Fred Richman,

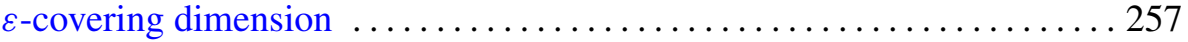

Thomas Curtis Craven and George Leslie Csordas, An inequality for the distribution of zeros of polynomials and entire functions $\ldots \ldots \ldots \ldots 263$

Thomas Jones Enright and R. Parthasarathy, The transfer of invariant

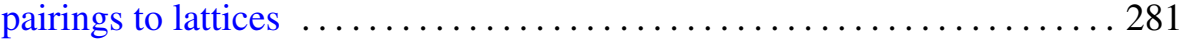

Allen Roy Freedman and John Joseph Sember, Densities and

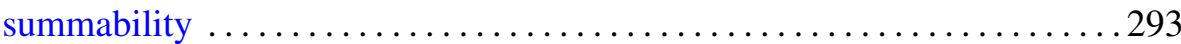

Robert Heller and Francis Aubra Roach, A generalization of a classical necessary condition for convergence of continued fractions . . . . . . 307

Peter Wilcox Jones, Ratios of interpolating Blaschke products ........... 311

V. J. Joseph, Smooth actions of the circle group on exotic spheres ........ 323

Mohd Saeed Khan, Common fixed point theorems for multivalued

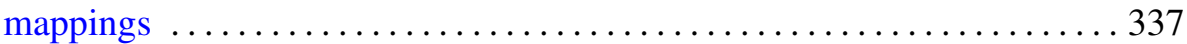

Samuel James Lomonaco, Jr., The homotopy groups of knots. I. How to

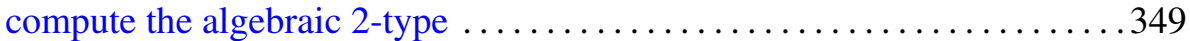

Louis Magnin, Some remarks about $C^{\infty}$ vectors in representations of connected locally compact groups ............................ 391

Mark Mandelker, Located sets on the line . . . . . . . . . . . . . . . . . 401

Murray Angus Marshall and Joseph Lewis Yucas, Linked quaternionic mappings and their associated Witt rings $\ldots \ldots \ldots \ldots \ldots \ldots \ldots \ldots . \ldots \ldots 11$

William Lindall Paschke, $K$-theory for commutants in the Calkin algebra

W. J. Phillips, On the relation $P Q-Q P=-i I$ 435

Ellen Elizabeth Reed, A class of Wallman-type extension. 443

Sungwoo Suh, The space of real parts of algebras of Fourier transforms 461 Antonius Johannes Van Haagen, Finite signed measures on function

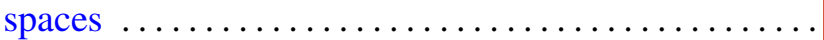

Richard Hawks Warren, Identification spaces and unique uniformity 